\title{
Une autre école en Espagne
}

Le projet Atlántida

A different school in Spain. The "Atlantida" project

Reformas educativas en España y otros modos de hacer escuela. El proyecto

"Atlántida"

\section{Antonio Bolívar}

Traducteur : Nuria Aligant

\section{OpenEdition}

\section{Journals}

Édition électronique

URL : http://journals.openedition.org/ries/447

DOI : $10.4000 /$ ries. 447

ISSN : 2261-4265

\section{Éditeur}

Centre international d'études pédagogiques

Édition imprimée

Date de publication : 1 décembre 2007

Pagination : 35-44

ISBN : 978-2-8542-0574-9

ISSN : $1254-4590$

Référence électronique

Antonio Bolívar, «Une autre école en Espagne », Revue internationale d'éducation de Sèvres [En ligne] 46 | décembre 2007, mis en ligne le 28 juin 2011, consulté le 19 avril 2019. URL : http:// journals.openedition.org/ries/447 ; DOI : 10.4000/ries.447 


\section{Le projet Atlántida*}

\section{Une autre école en Espagne}

\section{Antonio Bolívar}

Les vingt dernières années ont connu une période de macroréformes éducatives essentiellement articulées autour des programmes et de la scolarité obligatoire. La Loi organique générale du système éducatif de 1990 (LOGSE), élaborée et approuvée par le Parti socialiste, avait pour objectif d'étendre la scolarité obligatoire jusqu'à l'âge de seize ans et d'apporter une réponse éducative qui s'inscrive dans un cadre commun et lisible pour l'ensemble des élèves. Parmi ses objectifs principaux, on comptait une nouvelle organisation des cycles d'enseignement, la revalorisation et le développement de la formation professionnelle initiale et l'amélioration de la qualité de l'enseignement moyennant l'adaptation et la mise en ouvre des programmes par l'établissement scolaire (Bolívar \& Rodríguez Diéguez, 2002).

Mais, face aux enjeux éducatifs actuels (Bolívar 2007a), force est de constater que la répétition de réformes sur le mode top-down (du haut vers le bas) qui ont eu lieu en Espagne au cours de la seconde moitié du siècle dernier ont entraîné un désenchantement certain. Ces vagues de réformes successives ne parviennent pas à toucher de manière significative le noyau dur de l'enseignement, notamment les pratiques pédagogiques qui sont demeurées immuables. David Tyack et Larry Cuban (2000), qui ont analysé les réformes de l'enseignement survenues dans la société américaine au cours du siècle dernier, ont conclu à l'existence d'un fossé récurrent entre réformes politiques et pratiques pédagogiques. En fin de compte, disent-ils, les mécanismes de fonctionnement qui gouvernent la culture scolaire (appelés "grammaires scolaires») assimilent sans difficulté tout changement étranger à leurs propres règles, ce qui amène l'École à réformer les réformes extérieures qui demeurent, trop souvent, des discours purement rhétoriques qui légitiment le changement.

Par ailleurs, le contexte post-moderne qui tend à individualiser les problèmes éducatifs et sociaux renforce l'impact direct des réformes sur l'identité professionnelle des enseignants (Bolívar et al., 2005). Les transformations ne peuvent s'accomplir en négligeant les émotions et les sentiments des enseignants ainsi que leurs parcours professionnels. Des réformes imposées qui font fi des trajectoires professionnelles enseignantes et qui ne parviennent pas à les insérer dans des cadres plus attractifs ne peuvent engendrer de leur part que des sentiments négatifs et de la résistance. Vouloir à tout prix imposer des changements sans intégrer les attentes et le ressenti des enseignants équivaut à les introduire par une porte dérobée et, par voie de conséquence, les vouer à l'échec.

\footnotetext{
* Article traduit par Nuria Aligant.
} 
Face à la tendance actuelle à vouloir rationaliser et structurer à l'excès toute réforme éducative, il convient d'envisager de nouveau l'École comme le lieu stratégique d'un changement qui doit être généré par en bas. Tyack et Cuban affirment que l'un des moyens les plus pertinents de changer l'École consiste à la «transformer de l'intérieur», à partir du travail des enseignants, en l'encourageant. L'École, qui se trouve actuellement dans un contexte hostile et qui essaie de répondre aux défis posés par les différents environnements dans lesquels elle évolue, se doit de rassembler ses forces vives pour y parvenir.

\section{LA NOUVELLE École}

L'époque glorieuse des projets novateurs et de ce qu'il est convenu d'appeler en Espagne les «mouvements de rénovation pédagogique» (MRP), inhérents à l'optimisme qui régnait dans les années soixante-dix, a coïncidé avec la fin de la dictature. L'École et les équipes pédagogiques ont riposté à cet héritage par une effervescence de nouveaux modèles scolaires et de méthodes innovantes. Cependant, à mesure que les macroréformes structurelles étaient imposées, ces mouvements autonomes de rénovation se sont éteints peu à peu. Ces dernières années, alors que les différents projets récents de réformes éducatives et de gestion perdaient de leur crédibilité, les organisations éducatives ont poursuivi l'élaboration de projets, comme autant de moteurs pour «faire bouger» l'École. Ils misent sur une capacité interne au changement (tant des écoles que des organisations, des individus et des groupes) afin de la régénérer et d'apporter les améliorations éducatives souhaitées.

En lieu et place des stratégies bureaucratiques, verticales ou trop rationnelles, certains mouvements autonomes prônent l'émergence de dynamiques de changement qui rendent aux différents acteurs éducatifs une place prépondérante. Il s'agit d'associer tous les membres de la communauté éducative aux processus en marche en les impliquant dans des démarches qui rendent à l'École sa capacité à s'auto-rénover de manière durable, et qui entraînent un changement significatif des pratiques dans des projets d'amélioration de la qualité éducative. Ces initiatives devraient progressivement s'élargir à l'ensemble du système éducatif et avoir des répercussions directes sur les enseignements et les apprentissages. De la même façon, elles devraient encourager les dispositifs et mécanismes d'entraide, en créant des réseaux d'établissements qui partagent les mêmes principes novateurs.

Cette nouvelle vision de l'École se décline de façon plurielle et diverse. ${ }^{1}$ En tant que membre du comité scientifique du projet Atlántida et à partir de

1. Une initiative actuelle répertorie en ligne les différents mouvements et propositions d'innovation en Espagne : Innova. Educadores en Red [http://innova.usal.es]. C'est un projet du Forum pour l'éducation publique qui reçoit le soutien du ministère de l'Éducation espagnol. Il prévoit d'élargir la participation et d'organiser un grand congrès sur des expériences novatrices. 
mes interventions dans les écoles membres, je fonderai mon exposé sur les initiatives qui, dans un contexte de globalisation et de société de l'information, souhaitent reconstruire la communauté scolaire selon une approche locale, en partant du principe que l'École ne peut satisfaire à elle seule tous les besoins de formation de la société actuelle.

Établir des réseaux entre les établissements scolaires, les familles et les autres acteurs de la communauté renforce le tissu social et rend plus forte l'École dans l'objectif d'améliorer les enseignements qu'elle dispense. Chacun des membres partage également la responsabilité d'éduquer à la citoyenneté. Enfin, ces différents projets revendiquent l'existence d'une éducation et d'une École démocratiques, qui garantissent l'équité éducative et préparent à l'exercice d'une citoyenneté active (Bolívar, 2007b).

Ces différents projets partent du postulat que, dans l'espace éducatif élargi actuel, il n'est pas envisageable de circonscrire l'action éducative aux seuls établissements scolaires et de la maintenir à l'écart du reste de la société, comme s'il s'agissait d'un îlot. Elles plaident pour que l'acte éducatif scolaire soit étroitement associé à son environnement immédiat, notamment aux familles et aux municipalités. Éduquer à la citoyenneté n'est pas uniquement l'affaire des éducateurs et des enseignants mais bien l'objectif partagé de l'ensemble des acteurs et des instances sociales qui y sont associés. Récupérer le sens communautaire de l'éducation suppose de construire un "nouveau pacte éducatif» dans lequel chacun assume une responsabilité partagée.

Pour ce faire, le projet Atlántida, ainsi que d'autres initiatives pour le renouveau éducatif comme les "Communautés d'apprentissage ${ }^{2}$, se définit comme une proposition en faveur de l'élargissement de l'acte éducatif rassemblant les familles, la municipalité ou le quartier. Ces deux projets ont pour objectif le développement d'une pratique éducative nouvelle qui améliore la qualité de l'enseignement pour tous. Actuellement, ce projet est lié à un travail de dynamisation du tissu associatif et de la société civile afin que l'éducation de tous les citoyens, au sens large du terme, devienne une réalité au sein de la famille et de la ville. L'École, les familles, les services culturels, sociaux et municipaux sont appelés à emprunter un chemin partagé auquel sont également associés les services et les responsables de la vie scolaire, les conseillers d'orientation socioprofessionnelle et tous ceux qui interviennent dans les processus d'acquisition de valeurs citoyennes.

Le projet «Communautés d'apprentissage» se base sur l'expérience conduite dans l'École pour adultes de la Verneda-Sant Martí (Sánchez, 1999) et plaide résolument pour que les inégalités éducatives et culturelles soient comblées à partir d'une approche paritaire. En somme, il s'agit de construire des sociétés «dialogiques» capables d'apporter des réponses pertinentes aux

2. Outre les travaux cités, on peut consulter le site web : http://www.comunidadesdeaprendizaje.net/ 
défis qui interpellent nos sociétés de l'information. Dans cette nouvelle société, l'enseignement "accorde une place de choix au dialogue paritaire et intègre les voix de toute la communauté afin de développer un projet pluriel et participatif qui tienne compte du contexte social, historique et culturel des élèves » (Elboj et al., 2002, 27). Il s'agit d'établir une responsabilité partagée entre les enseignants, les élèves, les familles et l'ensemble de la communauté, qui encourage et développe les opportunités d'apprentissage pour tous les élèves. Les concertations, accords et alliances entre tous ces acteurs, notamment les familles, moyennant un dialogue paritaire, rendent possible la mise en œuvre avec succès de la mission éducative, a fortiori lorsqu'il s'agit de contextes défavorisés (Flecha, 1997).

Le projet «Communautés d'apprentissage» est implanté dans trentehuit établissements scolaires du premier et du second degré en Catalogne, au Pays Basque, en Aragon, ainsi que dans d'autres régions espagnoles. Dans certaines d'entre elles, il jouit du soutien des instances éducatives. Dans tous les établissements faisant partie du projet, la participation et l'engagement de l'ensemble des acteurs de la communauté éducative dans des initiatives qui améliorent les processus d'enseignement/apprentissage et les pratiques pédagogiques réussies sont vivement encouragés. Les méthodes d'organisation différenciées ainsi que le soutien aux élèves basés sur les cycles d'enseignement et la situation socioculturelle de chacun d'entre eux sont développés. Ce projet représente actuellement une de plus puissantes initiatives pour créer d'autres modèles d'École.

\section{RECONSTRUIRE LA COMMUNAUTÉ, UNE NOUVELLE APPROCHE LOCALE}

Une tradition séculaire, héritée de la modernité des Lumières, s'obstine à croire que les programmes d'enseignement sont la clé d'un changement réussi. Mais dans la conjoncture actuelle, il faut également agir sur l'idée de communauté si l'on veut situer l'enseignement dans la société de l'information. Ainsi, dans une société de l'information qui divise, dans des contextes familiaux déstructurés et avec des capitaux culturels hétérogènes, il faut orienter les efforts vers une approche qui prenne également en compte l'environnement immédiat, local. Accroître le capital social au service de l'éducation des citoyens suppose, en premier lieu, de le rattacher à l'action familiale et d'étendre ses domaines d'intervention à la ville, afin de répondre aux nouveaux défis sociaux. Famille, école et communauté constituent trois cercles qui, selon le niveau d'intersection et de recoupement, ont un effet certain sur l'éducation des élèves (Luengo, 2006).

Dans un contexte de mondialisation, reconstruire les communautés et les quartiers des grandes villes exige qu'on imagine des méthodes d'intervention nouvelles. Même s'il ne s'agit que d'une première étape, cette approche doit 
comporter non seulement le développement des liens avec les familles et l'environnement, mais ambitionner également la création de communautés locales composées de réseaux civiques susceptibles de représenter une réelle alternative aux processus globalisants. Autrement dit, la globalisation se construit d'en bas, elle est solidaire, fondée sur le dialogue et structurée autour de réseaux stratégiques (différents acteurs et institutions) dans chaque communauté. Par conséquent, agir localement est une réponse à la globalisation et "g-local» est le terme hybride choisi pour désigner la nécessité de constituer des communautés locales de réseaux solidaires qui construisent la globalisation d'en bas. En un sens, l'ancienne conscience de classe qui caractérisait la société industrielle est remplacée ici par la «conscience du lieu», en tant que nouvel espace public de construction de la citoyenneté.

La citoyenneté doit se forger localement tout d'abord, moyennant la création d'espaces de participation qui facilitent une mise en commun du travail. L'urbaniste Magnaghi (2003) a expliqué ce que signifie de nos jours un «projet local» qui implique, entre autres, la participation active de la société à sa construction. Une approche radicale de la démocratie dans le contexte actuel, suppose qu'elle s'exerce dans les environnements les plus immédiats, au niveau municipal ou dans les quartiers des grandes villes. Le mouvement actuel du nouveau "localisme» est né de cette idée, c'est-à-dire, en tant qu'espace où les politiques du bien-être, de l'éducation et des liens communautaires peuvent trouver toute leur expression.

L'Espagne ne possède qu'une tradition limitée en matière de territorialisation de l'éducation, par rapport à la municipalisation des pays anglo-saxons, puisque le rôle des municipalités y a été assez résiduel et périphérique. Cependant, à l'image de la plupart des pays occidentaux, le pays suit la tendance générale à la décentralisation et au transfert de compétences aux collectivités territoriales (Subirats, 2002). Depuis le premier congrès international des villes éducatrices, qui s'est tenu en 1990 à Barcelone, et la déclaration de la Charte des villes éducatrices, les projets développés par les municipalités se sont multipliés. Après avoir pris conscience de leur mission éducative, ces villes planifient des actions de développement de leurs ressources culturelles au bénéfice de l'éducation de tous leurs citoyens. En parallèle, les «Projets éducatifs de villes » connaissent un grand essor en tant que plans d'action (objectifs et axes de travail prioritaires des politiques éducatives dans le cadre de la ville et au bénéfice de la citoyenneté). Ces projets sont autant d'engagements pour conduire l'éducation au-delà des murs de l'École, par le biais de partenariats entre l'École et la communauté. Outre les ressources éducatives, l'environnement communautaire met également au service de la société ses espaces ainsi que d'autres acteurs de l'éducation, ce qui amène Subirats (2005, 193-194) à ce commentaire : «L'École, avec l'ensemble des acteurs qui participent aux processus éducatifs, doit trouver dans l'environnement immédiat et communautaire, dans la ville par exemple, le cadre essentiel 
dans lequel elle doit développer son travail, projeter toute sa puissance éducative et tirer bénéfice du potentiel qu'il lui offre, tout en rendant l'ensemble de ces acteurs co-responsables des problèmes communs ainsi que des possibles solutions.»

Enfin, les actions promues par le projet Atlántida s'inscrivent dans le cadre de ce large mouvement appelé «nouveau localisme». Les problèmes concernant l'éducation et l'éducation à la citoyenneté, au sens large, débordent du cadre scolaire et exigent que soient menées des actions conjointes entre l'établissement scolaire et son environnement communautaire.

\section{Le PROJet ATLÁNTIDA SUR L'ÉDUCATION À LA DÉMOCRATIE}

Le projet Atlántida regroupe un collectif hétérogène de professionnels de l'éducation (enseignants du premier et second degré et formateurs pour adultes, du supérieur, parents d'élèves et représentants des collectivités locales) qui constituent des groupes de travail reliés aux établissements scolaires et qui mènent des actions sur l'éducation à la démocratie et à la citoyenneté. Le projet est né des travaux de réflexion d'un groupe autonome des îles Canaries qui avait reçu le soutien de l'État à travers le mouvement pour l'innovation ADEME (association pour le développement et l'amélioration de l'École). En 1998 débute l'élaboration d'un cadre théorique, qui fixe les axes de travail pour une École démocratique et, postérieurement, développe des échanges d'expériences entre les établissements scolaires de plusieurs régions espagnoles : îles Canaries, Madrid, Etrémadure et Andalousie. Au cours de ces dernières années, le projet s'est étoffé, avec la parution de diverses publications et matériaux concernant les programmes d'enseignement, le soutien manifesté par plusieurs organismes et collectifs, ainsi que par un réseau d'établissements scolaires d'enseignement préscolaire, primaire, secondaire et d'adultes qui constitue le socle du projet.

Le projet Atlántida, plate-forme de travail pour l'amélioration de l'enseignement public, constitue une initiative ouverte à la participation de tous les acteurs éducatifs qui le souhaitent. Il a pour objectif le développement des idées et des valeurs inhérentes à une éducation à la démocratie basée sur les éléments suivants : garantir le droit à l'éducation de façon équitable à tous les élèves qui, au terme de la scolarité obligatoire, doivent avoir acquis un socle commun de connaissances leur permettant d'exercer leur citoyenneté sans risque d'exclusion sociale. Atteindre cet objectif exige qu'il soit partagé par le plus grand nombre d'enseignants, de parents d'élèves et, plus généralement, par tous les partenaires sociaux qui interviennent dans ces processus. Le projet s'inscrit dans la continuité du travail mené par les «écoles démocratiques » qui encouragent la participation des élèves et des parents d'élèves à la vie scolaire. Cette approche fait écho à celle de "programmes démocratiques» (Guarro, 2002) qui prônent un choix collégial et participatif du patrimoine culturel accessible à tous les élèves et garant de l'équité éducative, à travers l'acquisition d'un socle 
commun de compétences. Cela se concrétise ensuite à travers l'organisation des contenus, des établissements scolaires et des activités pédagogiques. ${ }^{3}$

Lors du séminaire sur le projet, le travail de réflexion s'est articulé autour de cinq axes principaux : création de bonnes écoles pour tous qui s'engagent dans des processus d'amélioration; élaboration d'un cadre théorique et pratique pour une éducation et une culture scolaires démocratiques; mise en place des conditions et des dispositifs pour améliorer la vie scolaire dans les établissements; mise en œuvre d'une éducation qui conduise à l'exercice d'une citoyenneté active et responsable; définition des contenus indispensables qui garantissent une éducation équitable à toute la société. Le projet est conscient des limites réelles auquel il est confronté, mais il propose également une utopie capable de mobiliser tous les acteurs et leur permettant d'atteindre les objectifs les plus ambitieux. Il prétend ainsi :

- analyser dans un esprit critique le socle culturel indispensable à notre société, et mettre l'accent sur une proposition qui renforce les valeurs démocratiques; il souhaite s'engager dans une rénovation du modèle socio-économique, socio-politique, socio-culturel et socio-affectif actuel, selon un axe de développement de l'éducation et de la citoyenneté démocratique et communautaire;

- développer des stratégies de soutien à la formation des enseignants et de conseil auprès des différentes instances qui prêtent main forte au système éducatif; elles permettront, à partir de problèmes concrets issus de la pratique, le développement et le soutien d'actions spécifiques dans les établissements scolaires et leur environnement susceptibles de favoriser la reconstruction démocratique de la culture scolaire tant au niveau du «vivre ensemble» que de la méthodologie ou de l'évaluation;

- soutenir et promouvoir des actions avec les parents d'élèves à travers les AMPAs ${ }^{4}$ et les services socio-éducatifs municipaux qui facilitent leur participation à la reconstruction démocratique de la culture scolaire; il s'agit du noyau pour le changement éducatif constitué par l'École, la Famille et la Municipalité réunis en "comités pour la citoyenneté»;

- en définitive, créer un réseau d'écoles démocratiques et constituer un capital d'expériences socio-éducatives menées dans un environnement proche, résolument engagées dans l'innovation démocratique des programmes scolaires obligatoires et tournées vers le modèle de société que l'on souhaite atteindre. L'objectif final tend à développer un réseau Atlántida dans différentes régions d'Espagne et de rejoindre ceux, similaires, qui existent déjà afin de défendre l'avenir du service public éducatif en proposant à l'État des expériences concrètes, par le biais d'un forum ou d'une plate-forme d'innovations démocratiques.

3. Voir la description de la vie scolaire de l'école La Navata à Feito (Guarro, 2006 : 117-180).

4. Asociaciones de Madres y de Padres de Alumnos (associations de parents d'élèves). 
Le projet Atlántida souhaite représenter une alternative novatrice aux missions qui incombent actuellement à l'École. Une éducation démocratique qui aspire à l'exercice de la citoyenneté active doit promouvoir la participation démocratique afin que l'exercice de celle-ci ait également lieu au sein de l'École. Aller au-delà du simple transfert des responsabilités éducatives aux établissements scolaires nécessite que la communauté éducative soit en mesure d'induire des processus de socialisation cohérents. Et c'est l'idée de communauté éducative intégrée dans un projet éducatif élargi qui conduit à parler de citoyenneté à responsabilité partagée. Il est évident que l'éducation à la démocratie et à la citoyenneté doit être le résultat de l'action conjuguée de plusieurs acteurs et institutions. De plus, afin que son fonctionnement soit cohérent, elle doit être partagée de façon coopérative par l'ensemble du tissu social. Dans le cas contraire, ses chances de réussite seront amoindries en raison de la faiblesse stratégique de la seule action scolaire.

Par ailleurs, en adoptant une voie engagée et critique, l'éducation au plein exercice de la citoyenneté doit faciliter la consolidation sociale de la démocratie en dotant les citoyens des connaissances et du savoir-faire nécessaires à une participation active à la politique. Ainsi, l'éducation démocratique acquiert tout son sens en tant que moyen de participation et de délibération dans les affaires courantes de la politique et elle se reflète dans des valeurs telles que la solidarité, la coopération, la justice, la tolérance ou le développement durable qui doivent faire partie des programmes scolaires. Le processus de transformation de l'École se concrétise à travers l'élaboration de programmes adaptés, ainsi que par le changement dans ses modes d'organisation qui permettent de vivre et d'apprendre les valeurs démocratiques.

\section{Parents d’élèves, ÉCOLES ET MUNICIPALITÉS}

Atlántida et son réseau d'écoles aspire à intégrer aussi bien la famille (écoles de parents d'élèves, associations de parents d'élèves) que le quartier (associations), les organisations non gouvernementales (actions éducatives conjointes) et la ville ( "Villes éducatrices», "Projets éducatifs de villes», "Pactes civiques» des mairies), afin de revigorer le tissu associatif et, par extension, celui de la société civile. La reconstruction d'une communauté - l'École certes, mais aussi la communauté éducative et sociale dans son ensemble - doit déboucher sur le développement de qualités civiques qui encouragent les bonnes pratiques citoyennes. Pour ce faire, Atlántida propose la création de comités de citoyenneté ou d'autres instances qui facilitent l'articulation entre école, famille et collectivités territoriales, en particulier municipales.

Afin de reprendre quelques-uns des axes les plus porteurs du changement éducatif, Atlántida propose la création de réseaux ainsi que la définition et la mise en œuvre d'accords ou de consortia regroupant écoles, parents d'élèves 
et représentants des municipalités. Ces actions apportent, selon les lieux où elles sont mises en œuvre, une valeur ajoutée et un potentiel certain de stratégies nouvelles qui devraient renforcer et améliorer les processus éducatifs. À travers les relations de confiance qui se tissent, les réseaux génèrent des engagements et des attentes réciproques de soutien mutuel ainsi qu'un capital d'information et de ressources dont l'éducation est la première bénéficiaire, en établissant des liens entre les divers acteurs et collectivités territoriales et en contribuant à développer le capital social dans chaque contexte. Créer des relations de confiance entre les familles, les établissements scolaires et plus généralement avec les citoyens, promouvoir l'échange d'informations et consolider tous ces liens à travers des réseaux, est un excellent moyen pour consolider le tissu social et la société civile. Seule la mobilisation de toutes les capacités sociales permettra d'améliorer l'éducation pour tous.

Au-delà de l'action menée dans les établissements scolaires, enseigner et apprendre à être citoyen passe par la construction d'une communauté éducative qui induise un processus de socialisation approprié. Atlántida crée des comités de citoyenneté là où le projet peut s'appuyer sur une articulation dynamique entre école, famille et services locaux municipaux. Par ailleurs, les actions dans le domaine de la citoyenneté doivent s'inscrire dans les structures et les dispositifs pédagogiques de l'établissement scolaire qui sera en mesure de garantir la meilleure articulation entre elles. Dans cet espace pédagogique élargi, des actions d'implication et de participation sociale des élèves (volontariat et services à la communauté et envers les institutions et les organisations) sont développées. Aujourd'hui, parler de citoyenneté est une plate-forme idéale pour explorer le modèle de société et d'éducation que nous voulons reconstruire et cela devrait nous conduire, à moyen terme, à aborder les thèmes que la dernière décennie a laissés en souffrance : quels programmes d'enseignement, quelles connaissances pour la société à reconstruire (Guarro, 2002)?

L'éducation à la citoyenneté est un objectif partagé et communautaire et pour l'atteindre, il faut que l'École sorte de son isolement afin que toute la communauté se co-responsabilise. L'École doit établir des pactes, des réseaux, des alliances avec les familles, les mairies et les services sociaux de la communauté dans laquelle elle évolue (Bolívar, 2007b). Dans le contexte complexe qui est le nôtre, des nouvelles formes de participation de la communauté éducative sont possibles. Le projet Atlántida propose des réseaux inter établissements scolaires, des accords avec les familles, les municipalités et d'autres acteurs de la communauté afin de consolider le tissu social, de renforcer "latéralement» la capacité d'amélioration de l'éducation des élèves. Ainsi, tous les acteurs se co-responsabilisent autour de l'éducation à la citoyenneté et dépassent la fragmentation des espaces et des temps éducatifs actuels. 


\section{BIBLIOGRAPHIE}

BOLÍVAR A. y RODRÍGUEZ DIÉGUEZ J.L. (2002) : Reformas y retórica. La reforma educativa de la LOGSE. Archidona (Málaga): Aljibe.

BOLÍVAR A. (2007a) : "Um ohlar actual sobre a mudança educativa: onde situar os esforços de melhoria?». In Carlinda Leite y Amelia Lopes (org.). Escola, currículo e formaçâo de identidades. Porto (Portugal): Edic. ASA, pp. 13-50.

BOLÍVAR A. (2007b): Educación para la ciudadanía. Algo más que una asignatura. Barcelona: Graó.

BOlílVAR A., GALlego M. J., LEÓN M. J. y PÉREZ P. (2005) : "Políticas educativas de reforma e identidades profesionales: El caso de la Educación Secundaria en España». Education Policy Analysis Archives, 13 (45). Disponible sur : [http://epaa.asu. edu/epaa/v13n45].

ELBOJ C. et al. (2002): Comunidades de aprendizaje. Transformar la educación. Barcelona. Graó.

ESCUDERO J.M. (2002): La reforma de la reforma. ¿Qué calidad, para quienes?. Barcelona: Ariel.

FEITO R. (2006) : Otra escuela es posible. Madrid: Siglo XXI.

FLECHA R. (1997): Compartiendo palabras. Teoría y práctica del aprendizaje dialógico. Barcelona: Paidós.

GUARRO A. (2002) : Currículum y democracia. Por un cambio de la cultura escolar. Barcelona. Octaedro.

LUENGO F. (2006) : «El Proyecto Atlántida: experiencias para fortalecer el eje escuela, familia y municipio ». Revista de Educación, 339, 177-194.

MAGNAGI A. (2003): Le projet local. Lieja-Bruselas: Mardaga.

SANCHEZ M. (1999): "La Verneda Sant Martí: a school where people dare to dream». Harvard Educational Review, 69 (3), pp. 320-335.

SUBIRATS J. (coord.) (2002) : Gobierno local y educación: la importancia del territorio y la comunidad en el papel de la escuela. Barcelona: Ariel.

SUBIRATS J. (2005): «Escuela y municipio. ¿Hacia unas nuevas políticas educativas locales? » In J. Gairín (coord.): La descentralización educativa. Barcelona. Praxis, pp. 177-207.

TYACK D. y CUBAN L. (2000): En busca de la utopía. Un siglo de reformas de las escuelas públicas. México: Fondo de Cultura Económica. 